\title{
Constructions of S-boxes with uniform sharing
}

\author{
Kerem Varici ${ }^{1}$, Svetla Nikova ${ }^{1}$, Ventzislav Nikov $^{2}$, and Vincent Rijmen ${ }^{1}$ \\ 1 KU Leuven, imec-COSIC, Belgium, \{name.surname\}@esat.kuleuven.be \\ 2 NXP Semiconductors, Belgium, venci.nikov@gmail.com
}

\begin{abstract}
In this paper we focus on S-box constructions. We consider the uniformity property of an S-box which plays an important role in Threshold Implementations (TI). Most papers so far have studied TI sharings for given S-boxes. We proceed in the opposite way: starting from $n$-bit S-boxes with known sharings we construct new $(n+1)$-bit S-boxes from them with the desired sharings. In addition, we investigate the self-equivalency of S-boxes and show some interesting properties.
\end{abstract}

\section{Introduction}

Block ciphers are among the key components in cryptography. Recently, lightweight cryptography became popular and it carried the design approach of a block cipher into different points. Depending on the requirements of the platform: low energy consumption, restriction on area, low multiplicative complexity, resistance against side-channel attacks etc., many new design approaches were proposed over the years.

\section{$1.1 \quad$ S-boxes}

In 1945, Shannon defined two properties of a block cipher: diffusion and confusion. Restrictions on lightweight cryptography push the search for perfectly secure diffusion and confusion components to find suboptimal secure but efficient components. S-boxes are among the main primitives in symmetric-key cryptography. They are the smallest component in an algorithm which provides non-linearity. The size of an S-box can change from three bits to $n$ bits but mostly lengths of four bits or eight bits are preferred.

In this work, we study general $n$-bit S-boxes but we do our experiments for small sizes i.e. three to five bits. The classification of all 3-bit and 4-bit S-boxes according to affine equivalency was first given in $[4,7]$.

Definition 1. Two $S$-boxes $S_{1}$ and $S_{2}$ are affine equivalent if there exists a pair of invertible affine permutations $A$ and $B$, such that $S_{1}=A \circ S_{2} \circ B$.

Note the ordering used in the paper for $A \circ B$ is first apply $A$, then apply $B$. It is well known that all invertible $2 \times 2 \mathrm{~S}$-boxes are affine, hence there is only one class. The set of invertible $3 \times 3$ S-boxes contains 4 equivalence classes: 3 classes containing quadratic functions, and one class containing the affine functions. The 
maximal algebraic degree of a balanced $n$-variable Boolean function is $n-1[5,8]$. De Cannière lists 302 equivalence classes for the $4 \times 4$ bijections: the class of affine functions, 6 classes containing quadratic functions and the remaining 295 classes containing cubic functions. There is a transformation [3] which expands the 3-bit classes $\mathcal{Q}_{1}^{3}, \mathcal{Q}_{2}^{3}$, and $\mathcal{Q}_{3}^{3}$ into $\mathcal{Q}_{4}^{4}, \mathcal{Q}_{12}^{4}$ and $\mathcal{Q}_{300}^{4}$ correspondingly. That is, given a 3 -bit permutation $S\left(x_{1}, x_{2}, x_{3}\right)=\left(y_{1}, y_{2}, y_{3}\right)$, its 4-bit extension is generated by $S\left(x_{1}, x_{2}, x_{3}, x_{4}\right)=\left(y_{1}, y_{2}, y_{3}, x_{4}\right)$.

Recently a classification of all quadratic $5 \times 5$ bijective S-boxes was presented in [1]. The authors have also pointed out that the 5 -bit classes $\mathcal{Q}_{1}^{5}, \mathcal{Q}_{3}^{5}, \mathcal{Q}_{4}^{5}, \mathcal{Q}_{7}^{5}, \mathcal{Q}_{13}^{5}$ and $\mathcal{Q}_{30}^{5}$ are extensions of the 4 -bit quadratic classes $\mathcal{Q}_{4}^{4}, \mathcal{Q}_{294}^{4}, \mathcal{Q}_{12}^{4}, \mathcal{Q}_{299}^{4}, \mathcal{Q}_{293}^{4}$ and $\mathcal{Q}_{300}^{4}$ from [3] respectively. That is, given a 4-bit permutation $S\left(x_{1}, x_{2}, x_{3}, x_{4}\right)=$ $\left(y_{1}, y_{2}, y_{3}, y_{4}\right)$, its 5 -bit extension is generated by $S\left(x_{1}, x_{2}, x_{3}, x_{4}, x_{5}\right)=$

$\left(y_{1}, y_{2}, y_{3}, y_{4}, x_{5}\right)$. Let $\bar{x}=\left(x_{1}, \ldots, x_{n}\right)$ then the method used in the above mentioned publications can be summarized as follows

$$
\begin{aligned}
S\left(\bar{x}, x_{n+1}\right) & =S_{1}(\bar{x}) \quad \text { for the first } n \text { bits } \\
& =x_{n+1} \quad \text { for the }(n+1) \text {-st bit }
\end{aligned}
$$

Another well known construction is the so-called Shannon expansion.

Definition 2 (Shannon Expansion). Let $\bar{x}=\left(x_{1}, \ldots, x_{n}\right)$ then

$$
F(\bar{x})=x_{i} F_{x_{i}}(\bar{x})+\left(x_{i}+1\right) F_{x_{i}+1}(\bar{x})
$$

where $F_{x_{i}}(\bar{x})=F\left(x_{1}, \ldots, x_{i}=1, \ldots, x_{n}\right)$ and $F_{x_{i}+1}(\bar{x})=F\left(x_{1}, \ldots, x_{i}=0, \ldots, x_{n}\right)$ i.e. these are two functions on $n-1$ variables $x_{1}, \ldots, x_{i-1}, x_{i+1}, \ldots, x_{n}$.

\subsection{Threshold implementations and uniform sharing}

The recent developments in the technology come with some additional security requirements like physical security. Today, many methods exist to construct physically secure implementations. One of the most popular ways is Threshold Implementations (TI) [10]. The method uses the idea of secret sharing schemes and techniques from multiparty computation, and requires a sharing which needs to satisfy the following properties [11]. Define a sharing for a value $a$ to be $\left(a^{1}, a^{2}, \cdots, a^{s}\right)$ satisfying $\sum_{i=1}^{s} a^{i}=a$.

Correctness: For all $y \in \mathbb{F}_{2}^{n}, y=F(x)$ implies that $y=\sum_{i=1}^{s} F^{i}\left(x^{1}, x^{2}, \cdots, x^{s}\right)$ for all sharings $\left(x^{1}, x^{2}, \cdots, x^{s}\right)$ of $x \in \mathbb{F}_{2}^{m}$.

Non-completeness: Every shared component function $F^{i}$ is independent of at least one share of the input variable $x$ to provide security against first order side channel attacks. This is often translated as $F^{i}$ should be independent of $x^{i}$.

Uniformity: For all sharings of $y$, the number of valid sharings $\left(x^{1}, x^{2}, \cdots, x^{s}\right)$ of $x$ for which $F^{j}\left(x^{1}, x^{2}, \cdots, x^{s}\right)=y^{j}, 1 \leq j \leq s$, is equal to $2^{(s-1)(m-n)}$ times the number of $x \in \mathbb{F}_{2}^{m}$ for which $y=F(x)$. In other words, the sharing preserves the output distribution. In particular, if $F$ is a permutation on $\mathbb{F}_{2}^{m}$ then the functions $\left(F^{1}, F^{2}, \cdots, F^{s}\right)$ define together a permutation on $\mathbb{F}_{2}^{m s}$. 
The number of shares in $d$-th order TI depends on the algebraic degree $t$ of the S-box - namely $s \geq t d+1$, hence for 1-st order security one needs at least $t+1$ shares. We do not consider here the approach based on decomposition on the S-box into low degree S-boxes.

It is well known how to find a uniform sharing for all $3 \times 3$ and $4 \times 4$ S-boxes [3]. Recall that a uniform sharing with 3 shares exists for $\mathcal{Q}_{1}^{3}, \mathcal{Q}_{2}^{3}$, but not for $\mathcal{Q}_{3}^{3}$; and a uniform sharing with 4,5 and more shares exists for all 3 of them. Also recall that a uniform sharing with 3 shares exists for $\mathcal{Q}_{4}^{4}, \mathcal{Q}_{294}^{4}, \mathcal{Q}_{12}^{4}, \mathcal{Q}_{299}^{4}, \mathcal{Q}_{293}^{4}$, but not for $\mathcal{Q}_{300}^{4}$; and a uniform sharing with 4,5 and more shares exists for all 6 of them $\mathcal{Q}_{4}^{4}, \mathcal{Q}_{294}^{4}, \mathcal{Q}_{12}^{4}, \mathcal{Q}_{299}^{4}, \mathcal{Q}_{293}^{4}$, and $\mathcal{Q}_{300}^{4}$. Also a uniform sharing with 4 shares is known for $\mathcal{C}_{1}^{4}, \mathcal{C}_{3}^{4}, \mathcal{C}_{13}^{4}$ and $\mathcal{C}_{301}^{4}$. The remaining cubic 4 -bit $\mathrm{S}$-boxes have no uniform sharing with 4 shares. However, all of them have a uniform sharing with 5 shares. Recently, a 3 -share uniform sharing for 30 of the 5-bit quadratic classes (namely classes $1-27,31,33$ and 34) was found [1]. Moreover, all 5-bit quadratic permutation classes have a uniform sharing with 4 and more shares. It is well known that any Boolean function of 3 or more variables has a uniform TI sharing with 3 and more sharings [11].

It is clear that method (1) is providing uniform sharing with $s$ shares for $S$ if and only if $S_{1}$ has a uniform sharing with $s$ shares. In this work, we focus on the uniformity property of an S-box since it needs a special treatment and there does not exist a straightforward way of checking the property. We show under which conditions a uniform sharing of $n$-bit bijective S-box can be used to construct uniform sharing of $(n+i)$-bit bijective S-boxes where $i \geq 1^{3}$. The main reason to do this, is that we want to avoid an exhaustive uniformity check, whose complexity increases exponentially with the size of the S-box and the number of shares used. We used the idea of Shannon's expansion to generate bigger S-boxes and manage to show the cases that uniformity will hold for the newly generated S-boxes. Moreover, we showed for small sizes $n=3$ and 4 , which $n$-bit classes maps to which $(n+1)$-bit classes.

The original intention of the affine equivalence algorithm [4] was to discover equivalence relations between different S-boxes, but the algorithm can be applied for a single S-box $S$ as well. In this case, the algorithm will return affine mappings $A$ and $B$ for the self-equivalent $S$. The number of different solutions for this equation can be seen as an indicator for the symmetry of the S-box [4]. S-boxes that have at least one non-trivial solution are called self-equivalent S-boxes [4].

A well known example is the 8-bit S-box $S$ used in RIJNDAEL [6]. It has 2040 different self-equivalence relations. Although this number might seem surprisingly high at first, in [4] it is shown that it can easily be explained from the special algebraic structure of the S-box of RIJNDAEL and it can be generalized for the inversion in $G F\left(2^{n}\right)$. Let first introduce the notation [a], which denotes the $n \times n$ bit matrix corresponding to a multiplication by $a$ in $G F\left(2^{n}\right)$. Similarly, denote by $Q$ the $n \times n$-bit matrix which performs the squaring operation in $G F\left(2^{n}\right)$.

\footnotetext{
3 The idea of constructing large S-boxes from smaller ones by considering the physical securirty is studied before in $[2,9]$. But none of these works focuses on uniformity property.
} 
Considering the fact that the RIJNDAEL S-box is defined as $S=i n v \circ A$ with $A$ a fixed affine mapping, we can derive a general expression for all pairs of affine mappings $C$ and $B$ that satisfy $B \circ S \circ C=S$

$$
C=A^{-1} \circ[a] \circ Q^{i} \circ A, \text { and } B=[a] \circ Q^{i}
$$

with $0 \leq i<n$ and $a \in G F\left(2^{n}\right) \backslash\{0\}$. Since $i$ takes $n$ different values and there are $2^{n}-1$ different choices for $a$, one obtains exactly 2040 in the case $n=8$ different solutions, while in general there are $n\left(2^{n}-1\right)$ self-equivalent S-boxes of the inversion. It is easy to check that $Q^{n}=I d$ and thus $Q^{i}=Q^{n-i}$.

As noted in [4] these ideas also apply to a large extent to other ciphers that use S-boxes based on power functions, e.g., CAMELLIA, MISTY and KASUMI, whose S-boxes $S 7$ and $S 9$ are both designed to be affine equivalent to a power function over $G F\left(2^{7}\right)$ and $G F\left(2^{9}\right)$, respectively. In this paper we will investigate this property for any S-box, i.e. not only for the power functions.

\subsection{Our contribution}

In Section 2 we first study the Shannon expansion for S-boxes and derive a condition to obtain a new invertible S-box. We then show how to use the Shannon expansion to construct from given $n$-bit bijective S-boxes with a uniform sharing $(n+1)$-bit bijective S-boxes with a uniform sharing and $(n+2)$-bit bijective S-boxes with a uniform sharing. We apply this for all 3-bit and 4-bit S-boxes to derive results on 4-bit and 5-bit S-boxes. Alas, we find that our method is not able to generate all $(n+1)$-bit bijective S-boxes. In Section 3 we present some results on the presence of self-equivalent S-boxes and involutions in affine equivalence classes. We conclude in Section 4.

\section{Constructions of S-boxes with uniform sharing}

\subsection{Increasing the size of an S-box by one}

We start with a slightly modified definition of Shannon's expansion.

Definition 3. Given two $n \times n$ S-boxes (bijections) $S_{1}(\bar{x})=\left(t_{1}, t_{2}, \ldots t_{n}\right)$ and $S_{2}(\bar{x})=\left(u_{1}, u_{2}, \ldots u_{n}\right)$, where $\bar{x}=\left(x_{1}, \ldots, x_{n}\right)$. Using Shannon's expansion, we get an $(n+1) \times(n+1) S$-box (not always a bijection) $S\left(x_{1}, \ldots, x_{n}, x_{n+1}\right)=$ $\left(y_{1}, y_{2}, \ldots, y_{n+1}\right)$ :

$$
\begin{aligned}
& y_{i}=x_{n+1} t_{i} \quad+\left(1+x_{n+1}\right) u_{i}, \quad \text { for } i=1, \ldots, n \\
& y_{n+1}=x_{n+1} F(\bar{x})+\left(1+x_{n+1}\right) G(\bar{x}),
\end{aligned}
$$

where $F$ and $G$ are Boolean functions of $n$ inputs.

It follows that the truth table of the constructed $(n+1) \times(n+1)$ S-box $S$ has the form shown in Table 1. 
Table 1. Truth table of $S$ constructed by Definition 3

\begin{tabular}{|l|l|}
\hline$\left(\bar{x}, x_{n+1}=0\right)$ & $\left(\bar{x}, x_{n+1}=1\right)$ \\
\hline$\left(S_{2}(\bar{x}), G(\bar{x})\right)$ & $\left(S_{1}(\bar{x}), F(\bar{x})\right)$ \\
\hline
\end{tabular}

Theorem 1. Given two $n \times n$ bijections $S_{1}$ and $S_{2}$, and an $(n+1) \times(n+1)$ $S$-box $S$ constructed via Shannon's expansion formula (3), then $S$ is a bijection if and only if

$$
G(\bar{x})=F\left(S_{1}^{-1}\left(S_{2}(\bar{x})\right)\right)+1 \quad \text { or equivalently } G=S_{2} \circ S_{1}^{-1} \circ F+1 \text { holds. }
$$

Proof. $(\Leftarrow)$ Let the condition $(4)$ hold and assume there are two different inputs $\left(\bar{x}^{\prime}, x_{n+1}^{\prime}\right)$ and $\left(\bar{x}^{\prime \prime}, x_{n+1}^{\prime \prime}\right)$, such that $S\left(\bar{x}^{\prime}, x_{n+1}^{\prime}\right)=S\left(\bar{x}^{\prime \prime}, x_{n+1}^{\prime \prime}\right)$, i.e. assume $S$ is not a bijection. Then we consider the following two cases:

a) if $x_{n+1}^{\prime}=x_{n+1}^{\prime \prime}$ then it follows that $\bar{x}^{\prime} \neq \bar{x}^{\prime \prime}$ since the inputs are different. From $S\left(\bar{x}^{\prime}, x_{n+1}^{\prime}\right)=S\left(\bar{x}^{\prime \prime}, x_{n+1}^{\prime \prime}\right)$ and $x_{n+1}^{\prime}=x_{n+1}^{\prime \prime}$, it follows that either $S_{1}\left(\bar{x}^{\prime}\right)=S_{1}\left(\bar{x}^{\prime \prime}\right)$ or $S_{2}\left(\bar{x}^{\prime}\right)=S_{2}\left(\bar{x}^{\prime \prime}\right)$. But since both $S_{1}$ and $S_{2}$ are bijections, we arrive at a contradiction.

b) if $x_{n+1}^{\prime} \neq x_{n+1}^{\prime \prime}$ then it follows either

b1) $S_{1}\left(\bar{x}^{\prime}\right) \stackrel{n+1}{=} S_{2}\left(\bar{x}^{\prime \prime}\right)$ and $F\left(\bar{x}^{\prime}\right)=G\left(\bar{x}^{\prime \prime}\right)$ or

b2) $S_{1}\left(\bar{x}^{\prime \prime}\right)=S_{2}\left(\bar{x}^{\prime}\right)$ and $F\left(\bar{x}^{\prime \prime}\right)=G\left(\bar{x}^{\prime}\right)$.

Let us consider case b1): since $S_{1}\left(\bar{x}^{\prime}\right)=S_{2}\left(\bar{x}^{\prime \prime}\right)$ we get $\bar{x}^{\prime}=S_{1}^{-1}\left(S_{2}\left(\bar{x}^{\prime \prime}\right)\right)$ and hence $F\left(S_{1}^{-1}\left(S_{2}\left(\bar{x}^{\prime \prime}\right)\right)\right)=G\left(\bar{x}^{\prime \prime}\right)$ but this contradicts to (4). Similarly one can get a contradiction for $\mathbf{b} \mathbf{2})$.

Therefore, our assumption is incorrect and hence $S$ is a bijection when the condition (4) holds.

$(\Rightarrow)$ Let $S$ be a bijection. Choose $x_{n+1}^{\prime}=0$ and $x_{n+1}^{\prime \prime}=1$, then for any $n$-tuple $\bar{x}^{\prime}$ there exist $n$-tuple $\bar{x}^{\prime \prime}$, such that $S_{1}\left(\bar{x}^{\prime}\right)=S_{2}\left(\bar{x}^{\prime \prime}\right)$. Since $S\left(\bar{x}^{\prime}, x_{n+1}^{\prime}\right) \neq S\left(\bar{x}^{\prime \prime}, x_{n+1}^{\prime \prime}\right)$ this implies that $F\left(\bar{x}^{\prime}\right) \neq G\left(\bar{x}^{\prime \prime}\right)$ or in other words $F\left(\bar{x}^{\prime}\right)=G\left(\bar{x}^{\prime \prime}\right)+1$. Again since $S_{1}\left(\bar{x}^{\prime}\right)=S_{2}\left(\bar{x}^{\prime \prime}\right)$ we get $\bar{x}^{\prime}=S_{1}^{-1}\left(S_{2}\left(\bar{x}^{\prime \prime}\right)\right)$ and thus $F\left(S_{1}^{-1}\left(S_{2}\left(\bar{x}^{\prime \prime}\right)\right)\right)+1=G\left(\bar{x}^{\prime \prime}\right)$ which is exactly the condition (4). This completes the proof.

We conclude that one has to choose only $S_{1}, S_{2}$ and $F$ in order to build $S$. When $S_{1}$ and $S_{2}$ are fixed there are "only" $2^{n}$ choices for $F$ in order to get $S$. We should stress that $\operatorname{deg}(S)=\max \{d A, d B\}$, where

$$
\begin{gathered}
d A= \begin{cases}\max \left\{\operatorname{deg}\left(S_{1}\right), \operatorname{deg}\left(S_{2}\right)\right\} & \operatorname{deg}\left(S_{1}+S_{2}\right)<\max \left\{\operatorname{deg}\left(S_{1}\right), \operatorname{deg}\left(S_{2}\right)\right\} \\
\max \left\{\operatorname{deg}\left(S_{1}\right), \operatorname{deg}\left(S_{2}\right)\right\}+1 & \operatorname{deg}\left(S_{1}+S_{2}\right)=\max \left\{\operatorname{deg}\left(S_{1}\right), \operatorname{deg}\left(S_{2}\right)\right\},\end{cases} \\
d B= \begin{cases}\max \{\operatorname{deg}(F), \operatorname{deg}(G)\} & \operatorname{deg}(F+G)<\max \{\operatorname{deg}(F), \operatorname{deg}(G)\} \\
\max \{\operatorname{deg}(F), \operatorname{deg}(G)\}+1 & \operatorname{deg}(F+G)=\max \{\operatorname{deg}(F), \operatorname{deg}(G)\} .\end{cases}
\end{gathered}
$$

Note that whenever $S_{1}=S_{2}$ condition (4) can be simplified to: $F(\bar{x})=G(\bar{x})+1$. In this case $\operatorname{deg}(S)=\max \left\{\operatorname{deg}\left(S_{1}\right), \operatorname{deg}(F)\right\}$, i.e. the simplified equation is:

$$
\begin{aligned}
y_{i} & =t_{i} & \text { for } i=1, \ldots, n \\
y_{n+1} & =x_{n+1}+F(\bar{x}) &
\end{aligned}
$$


Here, $t_{i}$ represents Boolean functions of $n$ inputs and $F$ is written instead of $F+1$ for simplicity. Note that compared to the construction (1) used in [3] to get from $3 \times 3$ an $4 \times 4$ S-box and similarly in [1] from $4 \times 4$ an $5 \times 5$ S-box, the construction (5) extends it to allow $F$ to be any Boolean function on $n$ variables.

Now we will show how the constructions (3) or (5) can be used to find uniform sharings. Let $\overline{1}_{i}$ denote the vector $(0, \ldots, 0,1,0, \ldots, 0)$ with 1 on the $i$-th position.

Theorem 2. Let us consider any $n \times n$ bijective $S$-box $S_{1}$ which has a uniform sharing with s shares and any Boolean function $F$ with $n$ variables which also has a uniform sharing with $s$ shares. If $S_{2}$ is chosen in one of the $n+1$ forms: $S_{1}(\bar{x})$, $S_{1}\left(\bar{x}+\overline{1}_{i}\right)$ for $i=1, \ldots, n$, then the generated via Theorem $1(n+1) \times(n+1)$-bit $S$-box $S$ by using $S_{1}, S_{2}$ and $F$ has also a uniform sharing with s shares.

Proof. When $S_{1}=S_{2}$ it follows from Theorem 1 that $S$ is a bijection if and only if $F(\bar{x})=G(\bar{x})+1$ and in this case the equation (5) can be used. It is also clear in this case that $S$ will have a uniform sharing with $s$ shares. Now let us consider the general case when $S_{2}(\bar{x})=S_{1}\left(\bar{x}+\overline{1}_{i}\right)$. According to Definition 2 we have:

$$
\begin{aligned}
S_{1}(\bar{x}) & =x_{i} S_{1, x_{i}}(\bar{x})+\left(x_{i}+1\right) S_{1, x_{i}+1}(\bar{x}) \\
F(\bar{x}) & =x_{i} F_{x_{i}}(\bar{x})+\left(x_{i}+1\right) F_{x_{i}+1}(\bar{x})
\end{aligned}
$$

We rewrite (6) as follows:

$$
\begin{aligned}
S_{1}(\bar{x}) & =\left(x_{i}+1\right)\left[S_{1, x_{i}}(\bar{x})+S_{1, x_{i}+1}(\bar{x})\right]+S_{1, x_{i}}(\bar{x}) \\
F(\bar{x}) & =\left(x_{i}+1\right)\left[F_{x_{i}}(\bar{x})+F_{x_{i}+1}(\bar{x})\right]+F_{x_{i}}(\bar{x})
\end{aligned}
$$

Note also that (6) implies:

$$
\begin{aligned}
S_{1}\left(\bar{x}+\overline{1}_{i}\right) & =\left(x_{i}+1\right) S_{1, x_{i}}(\bar{x})+x_{i} S_{1, x_{i}+1}(\bar{x}) \\
F\left(\bar{x}+\overline{1}_{i}\right) & =\left(x_{i}+1\right) F_{x_{i}}(\bar{x})+x_{i} F_{x_{i}+1}(\bar{x})
\end{aligned}
$$

Next we replace $S_{2}(\bar{x})$ with $S_{1}\left(\bar{x}+\overline{1}_{i}\right)$ in the definition of $S$

$$
\begin{array}{rlr}
S\left(\bar{x}, x_{n+1}\right) & =x_{n+1} S_{1}(\bar{x})+\left(1+x_{n+1}\right) S_{1}\left(\bar{x}+\overline{1}_{i}\right) \quad \text { for } i=1, \ldots, n \\
& =x_{n+1} F(\bar{x})+\left(1+x_{n+1}\right)\left[F\left(\bar{x}+\overline{1}_{i}\right)+1\right]
\end{array}
$$

Here we used that condition (4) should hold in order for $S$ to be a bijection. Then let's rewrite the first $n$-bits of (9) using (6) and (8)

$$
\begin{aligned}
S\left(\bar{x}, x_{n+1}\right)_{\mid 1 \ldots n} & =x_{n+1} S_{1}(\bar{x})+\left(1+x_{n+1}\right) S_{1}\left(\bar{x}+\overline{1}_{i}\right) \\
& =x_{n+1} x_{i} S_{1, x_{i}}(\bar{x})+x_{n+1}\left(x_{i}+1\right) S_{1, x_{i}+1}(\bar{x}) \\
& +\left(1+x_{n+1}\right)\left(x_{i}+1\right) S_{1, x_{i}}(\bar{x})+\left(1+x_{n+1}\right) x_{i} S_{1, x_{i}+1}(\bar{x}) \\
& =\left[x_{n+1}+x_{i}+1\right] S_{1, x_{i}}(\bar{x})+\left[x_{n+1}+x_{i}\right] S_{1, x_{i}+1}(\bar{x}) \\
& =\left[x_{n+1}+x_{i}\right]\left[S_{1, x_{i}}(\bar{x})+S_{1, x_{i}+1}(\bar{x})\right]+S_{1, x_{i}}(\bar{x})
\end{aligned}
$$

Comparing (10) with (7), we notice that the change of variables, namely $\left[x_{n+1}+x_{i}\right]$ to $\left[x_{i}+1\right]$ gives a equivalence between the first $n$ bits of $S$ and $S_{1}$. Since (7) has 
a uniform sharing then (10) also has a uniform sharing, meaning that this is an $s n \times=s n$ bijection. Finally, let's rewrite the last $(n+1)$-st bit of (9) using (6) and (8)

$$
\begin{aligned}
S\left(\bar{x}, x_{n+1}\right)_{\mid n+1} & =x_{n+1} F(\bar{x})+\left(1+x_{n+1}\right)\left[F\left(\bar{x}+\overline{1}_{i}\right)+1\right] \\
& =x_{n+1} x_{i} F_{x_{i}}(\bar{x})+x_{n+1}\left(x_{i}+1\right) F_{x_{i}+1}(\bar{x}) \\
& +\left(1+x_{n+1}\right)\left(x_{i}+1\right) F_{x_{i}}(\bar{x})+\left(1+x_{n+1}\right) x_{i} F_{x_{i}+1}(\bar{x})+\left(1+x_{n+1}\right) \\
& =\left[x_{n+1}+x_{i}+1\right] F_{x_{i}}(\bar{x})+\left[x_{n+1}+x_{i}\right] F_{x_{i}+1}(\bar{x})+\left(1+x_{n+1}\right) \\
& =\left[x_{n+1}+x_{i}\right]\left[F_{x_{i}}(\bar{x})+F_{x_{i}+1}(\bar{x})\right]+F_{x_{i}}(\bar{x})+\left(1+x_{n+1}\right)
\end{aligned}
$$

Now comparing (11) with (7) and using again the change of variables, namely $\left[x_{n+1}+x_{i}\right]$ to $\left[x_{i}+1\right]$, it gives "near" equivalence between the last bit of $S$ and $F$ except the term $x_{n+1}+1$. In other words, $S$ can be rewritten as follows:

$$
\begin{aligned}
S_{j}\left(\bar{x}, x_{n+1}\right) & =S_{1 j}(\bar{x}) & \text { for } j=1, \ldots, n \\
& =x_{n+1}+F(\bar{x})+1 &
\end{aligned}
$$

upon the change of variables $\left[x_{n+1}+x_{i}\right]$ to $\left[x_{i}+1\right]$. This completes the proof, since this is equivalent to the case $S_{2}=S_{1}$ where we know uniform sharings exist.

To summarize: the case $S_{2}(\bar{x})=S_{1}\left(\bar{x}+\overline{1}_{i}\right)$ reduces to the case $S_{2}(\bar{x})=S_{1}(\bar{x})$ with the change of variables $\left[x_{n+1}+x_{i}\right]$ to $\left[x_{i}+1\right]$.

\subsection{Application of Shannon's Expansion to S-boxes}

We first investigate what happens when we use constructions (3) or (5) with affine equivalent bijective S-boxes. Let $S$ be constructed from $S_{1}, S_{2}, F$ and $S^{\prime}$ be constructed from $S_{1}^{\prime}=A \circ S_{1} \circ B, S_{2}^{\prime}=A \circ S_{2} \circ B$ and $F^{\prime}=A \circ F$. Then we have:

$$
\begin{aligned}
S\left(\bar{x}, x_{n+1}\right) & =x_{n+1} S_{1}(\bar{x})+\left(1+x_{n+1}\right) S_{2}(\bar{x}) \\
& =x_{n+1} F(\bar{x})+\left(1+x_{n+1}\right) G(\bar{x}),
\end{aligned}
$$

where $G(\bar{x})=S_{2} \circ S_{1}^{-1} \circ F(\bar{x})+1$ and

$$
\begin{aligned}
S^{\prime}\left(\bar{x}, x_{n+1}\right) & =x_{n+1}[A \circ S 1 \circ B(\bar{x})]+\left(1+x_{n+1}\right)\left[A \circ S_{2} \circ B(\bar{x})\right] \\
& =x_{n+1}[A \circ F(\bar{x})]+\left(1+x_{n+1}\right) G^{\prime}(\bar{x}) .
\end{aligned}
$$

Since $S_{1}^{-1}=B^{-1} \circ S_{1}^{-1} \circ A^{-1}$ we have

$$
G^{\prime}=\left[A \circ S_{2} \circ B\right] \circ\left[B^{-1} \circ S_{1}^{-1} \circ A^{-1}\right] \circ[A \circ F]=A \circ S_{2} \circ S_{1}^{-1} \circ F=A \circ G .
$$

If we define $A^{\prime}=\left[\begin{array}{ll}A & 0 \\ 0 & 1\end{array}\right]$ and $B^{\prime}=\left[\begin{array}{ll}B & 0 \\ 0 & 1\end{array}\right]$ then we see that $A^{\prime} \circ S \circ B^{\prime}=S^{\prime}$ i.e. the constructed $S$ and $S^{\prime}$ are affine equivalent.

We will now explore two approaches that use this affine equivalence of the extended S-box to reduce the search complexity. 
1. We go class per class by first fixing $S_{1}=S_{2}$ to the class representative and then vary $F$ over all possible Boolean functions to get $S$ in its reduced form via the construction (5).

2. We go again class per class by first fixing $S_{1}$ to the class representative. However, next we run a second loop for $S_{2}$ varying it over all possible bijective S-boxes and last we vary $F$ over all possible Boolean functions. Then $S$ is obtained in its most general form via the construction (3).

Applying the first approach over $3 \times 3$ S-boxes to obtain $4 \times 4$ S-boxes showed that (5) enriches the number of classes we can construct and the uniform sharings we can obtain as shown in Table 2 .

Table 2. Extension of 3-bit S-box classes into 4-bit S-box classes

\begin{tabular}{l|lll}
\hline 3-bit Class & \multicolumn{2}{|l}{ 4-bit Class } \\
\hline $\mathcal{A}_{0}^{3}$ & $\mathcal{A}_{0}^{4}$, & $\mathcal{C}_{1}^{4}$, & $\mathcal{Q}_{4}^{4}$ \\
$\mathcal{Q}_{1}^{3}$ & $\mathcal{C}_{3}^{4}$, & $\mathcal{Q}_{4}^{4}$, & $\mathcal{Q}_{294}^{4}$ \\
$\mathcal{Q}_{2}^{3}$ & $\mathcal{C}_{13}^{4}$, & $\mathcal{Q}_{12}^{4}$, & $\mathcal{Q}_{293}^{4}$ \\
$\mathcal{Q}_{3}^{3}$ & $\mathcal{C}_{301}^{4}$, & $\mathcal{Q}_{300}^{4}$ &
\end{tabular}

These results show that in addition to the four corresponding classes $\mathcal{A}_{0}^{4}, \mathcal{Q}_{4}^{4}$, $\mathcal{Q}_{12}^{4}$ and $\mathcal{Q}_{300}^{4}$ which were already known from [3], we also get three additional quadratic classes and even four cubic classes. Using Theorem 2 we get a uniform sharing with $s$ shares for $S$, whenever such sharing exists for $S_{1}$ and $F$. This explains the results obtained in [3] for the four cubic S-boxes which are the only ones among the cubic $4 \times 4$ S-boxes, which have uniform sharing with 4 -shares. However, it also should be noted that we were not able to obtain class $\mathcal{Q}_{299}^{4}$ from any of the $3 \times 3$ S-boxes via this approach.

Similarly, applying the first approach over $4 \times 4$ S-boxes to obtain $5 \times 5$ but restricted only to the affine and quadratic S-boxes gives the results shown in Table 3. We obtain 23 out of the 75 quadratic classes given in [1]. In addition, it is clear from this construction why for the classes $\mathcal{Q}_{30}^{5}$ and $\mathcal{Q}_{32}^{5}$ no uniform sharing with 3 shares was found in [1]. Namely, they are extensions of class $\mathcal{Q}_{300}^{4}$ which has no uniform sharing itself.

We finish this section by applying the second approach over $3 \times 3$ S-boxes to obtain $4 \times 4$ S-boxes. It is to be expected that the second approach based on the construction (3) generates more solutions than the first one based on construction (5), however, the complexity is much higher. We experimentally determined that by constructing 4-bit S-boxes from 3-bit S-boxes we obtain all the 4-bit classes except the 11 classes presented in Table 4 . Notice that 8 out of these 11 exceptions belong to the Optimal Golden S-boxes [7]. Recall that there are 16 classes of the best 4-bit S-boxes i.e., $\{S: \operatorname{Diff}(S)=4, \operatorname{Lin}(S)=8\}=\left\{G_{0}, . ., G_{15}\right\}$ and among them is the inversion $G_{3}$. Apparently the Shannon expansion has certain limitations, which make it impossible to obtain the 11 classes from Table 4. For one example, namely the class $\mathcal{Q}_{193}^{4}$, we did some kind of backward search 
Table 3. Extension of non-cubic 4-bit S-box classes into 5-bit S-box classes

\begin{tabular}{l|l}
\hline 4-bit Class & 5 -bit Class \\
\hline $\mathcal{A}_{0}^{4}$ & $\mathcal{Q}_{0}^{5}, \mathcal{Q}_{1}^{5}, \mathcal{Q}_{14}^{5}$ \\
$\mathcal{Q}_{4}^{4}$ & $\mathcal{Q}_{1}^{5}, \mathcal{Q}_{2}^{5}, \mathcal{Q}_{3}^{5}, \mathcal{Q}_{15}^{5}, \mathcal{Q}_{18}^{5}$ \\
$\mathcal{Q}_{12}^{4}$ & $\mathcal{Q}_{4}^{5}, \mathcal{Q}_{6}^{5}, \mathcal{Q}_{13}^{5}, \mathcal{Q}_{17}^{5}, \mathcal{Q}_{20}^{5}, \mathcal{Q}_{21}^{5}$ \\
$\mathcal{Q}_{293}^{4}$ & $\mathcal{Q}_{13}^{5}, \mathcal{Q}_{24}^{5}, \mathcal{Q}_{31}^{5}$ \\
$\mathcal{Q}_{294}^{4}$ & $\mathcal{Q}_{3}^{5}, \mathcal{Q}_{5}^{5}, \mathcal{Q}_{12}^{5}, \mathcal{Q}_{16}^{5}, \mathcal{Q}_{19}^{5}, \mathcal{Q}_{23}^{5}$ \\
$\mathcal{Q}_{299}^{4}$ & $\mathcal{Q}_{7}^{5}, \mathcal{Q}_{22}^{5}$ \\
$\mathcal{Q}_{300}^{4}$ & $\mathcal{Q}_{30}^{5}, \mathcal{Q}_{32}^{5}$ \\
\hline
\end{tabular}

to verify our results. For each S-box in this class we used (3) to determine all possible decompositions to 3-bit S-boxes. We tried with using each of the 4 variables $x_{1}, x_{2}, x_{3}, x_{4}$ as $x_{n+1}$ in the formula and used every row once as "the last row". In this way, for any 4-bit S-box we derived 16 cases and for each of them obtained two 3-bit S-boxes. We found that for none of the S-boxes in this class and none of the 16 cases subsequently considered the obtained 3-bit S-boxes are permutations. This confirms that $\mathcal{Q}_{193}^{4}$ cannot be obtained via (3) when $S_{1}$ and $S_{2}$ are permutations.

Table 4. 4-bit S-box classes not obtained from 3-bit S-box classes

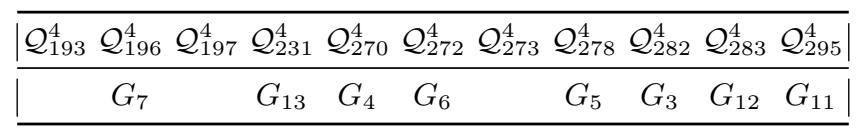

\subsection{Extending an S-box with two dimensions}

Given four $n \times n$ S-boxes (bijections) $S_{1}(\bar{x})=\left(t_{1}, t_{2}, \ldots t_{n}\right), S_{2}(\bar{x})=\left(u_{1}, u_{2}, \ldots u_{n}\right)$, $S_{3}(\bar{x})=\left(v_{1}, v_{2}, \ldots v_{n}\right)$ and $S_{4}(\bar{x})=\left(w_{1}, w_{2}, \ldots w_{n}\right)$, where $\bar{x}=\left(x_{1}, \ldots, x_{n}\right)$ then using Shannon's expansion we get an $(n+2) \times(n+2)$ S-box (not always a bijection) $S\left(x_{1}, \ldots, x_{n+1}, x_{n+2}\right)=\left(y_{1}, y_{2}, \ldots, y_{n+2}\right)$ with

$$
\begin{gathered}
y_{i}=x_{n+2}\left[x_{n+1} t_{i}+\left(1+x_{n+1}\right) u_{i}\right] \\
+\left(1+x_{n+2}\right)\left[x_{n+1} v_{i}+\left(1+x_{n+1}\right) w_{i}\right], \text { for } i=1, \ldots, n \\
y_{n+1}=x_{n+2}\left[x_{n+1} F_{1}(\bar{x})+\left(1+x_{n+1}\right) G_{1}(\bar{x})\right] \\
+\left(1+x_{n+2}\right)\left[x_{n+1} F_{2}(\bar{x})+\left(1+x_{n+1}\right) G_{2}(\bar{x})\right] \\
y_{n+2}=x_{n+2}\left[x_{n+1} F_{3}(\bar{x})+\left(1+x_{n+1}\right) G_{3}(\bar{x})\right] \\
+\left(1+x_{n+2}\right)\left[x_{n+1} F_{4}(\bar{x})+\left(1+x_{n+1}\right) G_{4}(\bar{x})\right]
\end{gathered}
$$

where $F_{1}, F_{2}, F_{3}, F_{4}$ and $G_{1}, G_{2}, G_{3}, G_{4}$ are Boolean functions of $n$ inputs. It follows from this definition that the truth table of the constructed $(n+2) \times(n+2)$ S-box $S$ has the following form. 
Table 5. Truth table of $S$

\begin{tabular}{|l|l|l|l|}
\hline $\mid\left(\bar{x}, x_{n+1}=0, x_{n+2}=0\right)$ & $\mid\left(\bar{x}, x_{n+1}=0, x_{n+2}=1\right)$ & $\mid\left(\bar{x}, x_{n+1}=1, x_{n+2}=0\right)$ & $\mid\left(\bar{x}, x_{n+1}=1, x_{n+2}=1\right)$ \\
\hline $\mid\left(S_{4}(\bar{x}), G_{2}(\bar{x}), G_{4}(\bar{x})\right)$ & $\left(S_{3}(\bar{x}), F_{2}(\bar{x}), F_{4}(\bar{x})\right)$ & $\left(S_{2}(\bar{x}), G_{1}(\bar{x}), G_{3}(\bar{x})\right)$ & $\left(S_{1}(\bar{x}), F_{1}(\bar{x}), F_{3}(\bar{x})\right)$ \\
\hline
\end{tabular}

Theorem 3. Given four $n \times n$ bijections $S_{1}, S_{2}, S_{3}$ and $S_{4}$ and an $(n+2) \times(n+2)$ $S$-box $S$ constructed via Shannon's expansion formula with two dimensions (13), then $S$ is a bijection if and only if

$$
\begin{aligned}
& F_{1}\left(S_{1}^{-1}(\bar{x})\right)=G_{2}\left(S_{4}^{-1}(\bar{x})\right)+1=F_{2}\left(S_{3}^{-1}(\bar{x})\right)=G_{1}\left(S_{2}^{-1}(\bar{x})\right)+1 \quad \text { and } \\
& F_{3}\left(S_{1}^{-1}(\bar{x})\right)=G_{4}\left(S_{4}^{-1}(\bar{x})\right)+1=F_{4}\left(S_{3}^{-1}(\bar{x})\right)+1=G_{3}\left(S_{2}^{-1}(\bar{x})\right) \quad \text { hold. }
\end{aligned}
$$

Proof (Sketch). The proof is similar to the proof of Theorem 1. First we consider the following sub cases separately: $x_{n+1}=0$ and $x_{n+2}=0,1 ; x_{n+1}=1$ and $x_{n+2}=0,1 ; x_{n+2}=0$ and $x_{n+1}=0,1 ; x_{n+2}=0$ and $x_{n+1}=0,1$. From those four sub cases we get four conditions accordingly:

$$
\begin{aligned}
G_{1}(\bar{z}) & =F_{1}\left(S_{1}^{-1}\left(S_{2}(\bar{z})\right)\right)+1 \\
G_{2}(\bar{z}) & =F_{2}\left(S_{3}^{-1}\left(S_{4}(\bar{z})\right)\right)+1 \\
G_{4}(\bar{z}) & =G_{3}\left(S_{2}^{-1}\left(S_{4}(\bar{z})\right)\right)+1 \\
F_{4}(\bar{z}) & =F_{3}\left(S_{1}^{-1}\left(S_{3}(\bar{z})\right)\right)+1
\end{aligned}
$$

or analogously (15) can be rewritten as:

$$
\begin{aligned}
G_{1}\left(S_{2}^{-1}(\bar{z})\right) & =F_{1}\left(S_{1}^{-1}(\bar{z})\right)+1 \\
G_{2}\left(S_{4}^{-1}(\bar{z})\right) & =F_{2}\left(S_{3}^{-1}(\bar{z})\right)+1 \\
G_{4}\left(S_{4}^{-1}(\bar{z})\right) & =G_{3}\left(S_{2}^{-1}(\bar{z})\right)+1 \\
F_{4}\left(S_{3}^{-1}(\bar{z})\right) & =F_{3}\left(S_{1}^{-1}(\bar{z})\right)+1
\end{aligned}
$$

Next consider the special case when the first n-bits $\bar{z}$ are fixed

$$
\begin{aligned}
& \bar{z}=S_{1}(\bar{d}) \\
& \bar{z}=S_{2}(\bar{c}) \\
& \bar{z}=S_{3}(\bar{b}) \\
& \bar{z}=S_{4}(\bar{a})
\end{aligned}
$$

and thus we have four different $n$-bit inputs $\bar{a}, \bar{b}, \bar{c}, \bar{d}$ as given above. Then the four tuples of the last two bits are formed by

$$
\begin{array}{r}
G_{2}(\bar{a}), G_{4}(\bar{a}) \\
F_{2}(\bar{b}), F_{4}(\bar{b}) \\
G_{1}(\bar{c}), G_{3}(\bar{c}) \\
F_{1}(\bar{d}), F_{3}(\bar{d})
\end{array}
$$


and we want to ensure that they are exactly the following four tuples $(0,0),(0,1),(1,0)$ and $(1,1)$ in any order. From this, four additional conditions can be derived

$$
\begin{aligned}
G_{2}(\bar{a}) & =F_{1}(\bar{d})+1 \\
G_{4}(\bar{a}) & =F_{3}(\bar{d})+1 \\
G_{1}(\bar{c}) & =F_{2}(\bar{b})+1 \\
G_{3}(\bar{c}) & =F_{4}(\bar{b})+1
\end{aligned}
$$

Combined with the previous four conditions namely (16) written in a similar way

$$
\begin{aligned}
G_{1}(\bar{c}) & =F_{1}(\bar{d})+1 \\
G_{2}(\bar{a}) & =F_{2}(\bar{b})+1 \\
G_{4}(\bar{a}) & =G_{3}(\bar{c})+1 \\
F_{4}(\bar{b}) & =F_{3}(\bar{d})+1
\end{aligned}
$$

we arrive at:

$$
\begin{aligned}
& F_{1}(\bar{d})=G_{2}(\bar{a})+1=F_{2}(\bar{b})=G_{1}(\bar{c})+1 \\
& F_{3}(\bar{d})=G_{4}(\bar{a})+1=F_{4}(\bar{b})+1=G_{3}(\bar{c})
\end{aligned}
$$

which is exactly the condition (14) in the Theorem. This completes the proof.

Therefore one has to choose only $S_{1}, S_{2}, S_{3}, S_{4}$ and $F_{1}, F_{3}$ in order to build $S$. When $S_{1}, S_{2}, S_{3}$ and $S_{4}$ are fixed there are $2^{n+1}$ choices for $F_{1}, F_{3}$ in order to get $S$. In the general case the construction then becomes as follows:

$$
\begin{aligned}
y_{i}=x_{n+2}[ & \left.x_{n+1} t_{i}+\left(1+x_{n+1}\right) u_{i}\right] \\
& +\left(1+x_{n+2}\right)\left[x_{n+1} v_{i}+\left(1+x_{n+1}\right) w_{i}\right] \text { for } i=1, \ldots, n \\
y_{i+1}=x_{n+2}[ & \left.x_{n+1} F_{1}(\bar{x})+\left(1+x_{n+1}\right)\left[F_{1}\left(S_{1}^{-1}\left(u_{i}\right)\right)+1\right]\right] \\
& +\left(1+x_{n+2}\right)\left[x_{n+1}\left[F_{1}\left(S_{1}^{-1}\left(v_{i}\right)\right)\right]+\left(1+x_{n+1}\right)\left[F_{1}\left(S_{1}^{-1}\left(w_{i}\right)\right)+1\right]\right] \\
y_{i+2}=x_{n+2}[ & \left.x_{n+1} F_{3}(\bar{x})+\left(1+x_{n+1}\right)\left[F_{3}\left(S_{1}^{-1}\left(u_{i}\right)\right)\right]\right] \\
& +\left(1+x_{n+2}\right)\left[x_{n+1}\left[F_{3}\left(S_{1}^{-1}\left(v_{i}\right)\right)+1\right]+\left(1+x_{n+1}\right)\left[F_{3}\left(S_{1}^{-1}\left(w_{i}\right)\right)+1\right]\right]
\end{aligned}
$$

The complexity to construct all $6 \times 6$ S-boxes given four $4 \times 4$ S-boxes is $2^{32}$.

\subsection{Applications of the extension with two dimensions}

If we rewrite the equation (13) for $y_{i}$ for $i=1, \ldots, n$ we get:

$$
y_{i}=x_{n+2} x_{n+1}\left(t_{i}+u_{i}+v_{i}+w_{i}\right)+x_{n+2}\left(u_{i}+w_{i}\right)+x_{n+1}\left(v_{i}+w_{i}\right)+w_{i}
$$

The case $n=3$ : We first focus on the case to construct quadratic 5-bit permutations given four quadratic 3 -bit permutations. Since the result has to be a quadratic permutation then $\left(t_{i}+u_{i}+v_{i}+w_{i}\right)=c$ where $c$ is constant and 
can be equal to zero. Note that if the quadruple $\left(t_{i}, u_{i}, v_{i}, w_{i}\right)$ is in the following formats $(\mathrm{X}, \mathrm{Y}, \mathrm{X}, \mathrm{Y}),(\mathrm{X}, \mathrm{Y}, \mathrm{Y}, \mathrm{X})$ or $(\mathrm{X}, \mathrm{X}, \mathrm{Y}, \mathrm{Y})$ where $\mathrm{X}, \mathrm{Y}$ is chosen from the set:

$$
S^{4}=\{S(x), S(x+1), S(x+2), S(x+4)),
$$

then $\left(t_{i}+u_{i}+v_{i}+w_{i}\right)=0$ and we get $y_{i}=x_{n+2}\left(u_{i}+w_{i}\right)+w_{i}$ or $y_{i}=$ $x_{n+1}\left(v_{i}+w_{i}\right)+w_{i}$ and guarantee the quadratic permutation. The number of different $y_{i}$ 's that we can generate in this way is given by: 3 formats times 4 choices for $\mathrm{X}$ times 3 choices for $\mathrm{Y}$ plus the 4 cases where $\mathrm{X}=\mathrm{Y}$, which equals 40. This small number of obtained permutations shows the limitation of this approach. For $y_{n+1}$ and $y_{n+2}$ we have to try out all possible constant, linear and quadratic functions. The number of possibilities is given by:

$$
\left(\prod_{i=0}^{2} 2^{\left(\begin{array}{c}
n \\
i
\end{array}\right)}\right)^{2}
$$

Table 6 shows the number of possibilities for $n=5,6,7$-bit S-boxes.

Table 6. Number of possibilities for $y_{n+1}$ and $y_{n+2}$ for quadratic S-boxes generated using (13)

\begin{tabular}{lc}
\hline$n$-bit S-box & Possibilities \\
\hline 5 & $2^{32}$ \\
6 & $2^{44}$ \\
7 & $2^{58}$ \\
8 & $2^{74}$ \\
\hline
\end{tabular}

The more general case: Recall that our goal is to obtain $y_{i}$ to be a quadratic permutation. Let's assume $t_{i}, u_{i}, v_{i}, w_{i}$ are quadratic permutations and by using the equation (18) one arrives to the following relations:

$$
\begin{aligned}
t_{i}+u_{i}+v_{i}+w_{i} & =\text { const } \\
u_{i}+w_{i} & =\text { affine }_{1} \\
v_{i}+w_{i} & =\text { affine }_{2}
\end{aligned}
$$

where affine $_{1}$ and affine $_{2}$ are not necessarily permutations. From (20) one derives

$$
\begin{aligned}
& u_{i}=w_{i}+\text { affine }_{1} \\
& v_{i}=w_{i}+\text { affine }_{2} \\
& t_{i}=w_{i}+\text { const }+ \text { affine }_{1}+\text { affine }_{2}=w_{i}+\text { affine }_{3}
\end{aligned}
$$

where const $=$ affine $_{3}+$ affine $_{1}+$ affine $_{2}$. Thus condition (21) now guarantees that $y_{i}$ in equation (18) will be quadratic iff $t_{i}, u_{i}, v_{i}, w_{i}$ are quadratic permutations. 
For the general $n$-bit permutation case, once the permutation $w_{i}$ is chosen the freedom to obtain $u_{i}, v_{i}$ and $t_{i}$ is to choose $n \times(2 \times(n+1)+1)$ coefficients and ensure that $u_{i}, v_{i}$ and $t_{i}$ are permutations. The freedom to choose $w_{i}$ is $n \times((n+1)+n \times(n-1) / 2))$ coefficients and to ensure that it is a permutation. For example fixing $n=3$ and constructing 5 -bit quadratic S-boxes gives $2^{48}$ possibilities. Note that here we use all possible S-boxes not only of the form (19).

\section{Permutations and Self-Equivalence}

In this section we will consider only S-boxes which are bijections. If we rewrite Definition 1 as $A^{-1} \circ S=S \circ B$ we can say that one can "push" the affine S-box $B$ through $S$ (so moving it from "left to right") and obtain $A^{-1}$. We can thus associate with a self-equivalent S-box $S$ the pair $(A, B)$, where one of the pair entries completely determines the other one. Note that for a given S-box $S$ more than one pair $(A, B)$ can exist (here we do not consider the trivial case of $A=B=I d$ ) since $S=A \circ S \circ B$ can also be rewritten as $A^{-1} \circ S \circ B^{-1}=S$, for example. In other words when $(A, B)$ is a pair for $S$ then $\left(A^{-1}, B^{-1}\right)$ is a pair too. For given S-box $S$ define the set

$$
S E_{S}=\left\{\left\{A, B, A^{-1}, B^{-1}\right\} \quad \mid S=A \circ S \circ B \quad \text { and } \quad S=A^{-1} \circ S \circ B^{-1}\right\}
$$

Note that for a given $S$ any affine permutation of the set $\left\{A, B, A^{-1}, B^{-1}\right\}$ determines uniquely the rest, e.g. $A$ determines $B$ and hence $A^{-1}, B^{-1}$.

Lemma 1. The number of self-equivalent pairs $(A, B)$ is an affine invariant, i.e. for all $S$-boxes $S$ in a given affine equivalent class $\left|S E_{S}\right|$ is constant.

Proof. Let $S^{\prime}$ and $S$ be two S-boxes which are affine equivalent (i.e. belong to the same class), then there exist affine permutations $C$ and $D$, such that $S^{\prime}=C \circ S \circ D$ or equivalently $S=C^{-1} \circ S^{\prime} \circ D^{-1}$. Using Definition 1 it follows that $C^{-1} \circ S^{\prime} \circ D^{-1}=A \circ C^{-1} \circ S^{\prime} \circ D^{-1} \circ B$ or equivalently $S^{\prime}=$ $C \circ A \circ C^{-1} \circ S^{\prime} \circ D^{-1} \circ B \circ D$. Denote by $A^{\prime}=C \circ A \circ C^{-1}$ and by $B^{\prime}=D^{-1} \circ B \circ D$ we get $S^{\prime}=A^{\prime} \circ S^{\prime} \circ B^{\prime}$.

Definition 4. An $S$-box $S$ is called (affine) self-equivalent inverse if there exist affine permutations $A$ and $B$ such that $S^{-1}=A \circ S \circ B$ holds.

Note that the inverse S-box in general may not belong to the same affine class, e.g. because it has different algebraic degree, however when this is the case we can establish some interesting properties. Again, if we rewrite the definition above as $A^{-1} \circ S^{-1}=S \circ B$ we can say that one can "push" the affine S-box $B$ through $S$ (so moving it from "left to right") and obtain $A^{-1}$ for $S^{-1}$.

Similar to the self-equivalence case one can thus associate with a self-equivalent inverse S-box $S$ the pair $(A, B)$ where one of the pair entries completely determines the other. Note that for a given S-box $S$ more than one pair $(A, B)$ can exist (here we do not consider the trivial case of $A=B=I d$ and $S$ involution). Indeed observe that if $(A, B)$ is a pair for self-inverse $S$ then $(B, A)$ is a pair too. 
Since $S^{-1}=A \circ S \circ B$ can be rewritten as $A^{-1} \circ S^{-1} \circ B^{-1}=S$ we obtain that $B \circ S \circ A=S^{-1}$ holds. Therefore for given S-box $S$ define the set

$$
S E I_{S}=\left\{\{A, B\} \quad \mid \quad S^{-1}=A \circ S \circ B \quad \text { and } \quad S^{-1}=B \circ S \circ A\right\}
$$

Note that for a given $S$ any affine permutation of the set $\{A, B\}$ uniquely determines the other, e.g. $A$ determines $B$.

Lemma 2. The number of self-equivalent inverse pairs $(A, B)$ is an affine invariant, i.e. all for $S$-boxes $S$ in a given affine equivalent class $\left|S E I_{S}\right|$ is constant.

Proof. Let $S^{\prime}$ and $S$ be 2 S-boxes which are affine equivalent (i.e. belong to the same class) then there exist affine permutations $C, D$ such that $S^{\prime}=C \circ S \circ D$ or equivalently $S=C^{-1} \circ S^{\prime} \circ D^{-1}$ and thus $S^{-1}=D \circ S^{-1} \circ C$. Using Definition 4 it follows that $D \circ S^{\prime-1} \circ C=A \circ C^{-1} \circ S^{\prime} \circ D^{-1} \circ B$. or equivalently $S^{-1}=D^{-1} \circ A \circ C^{-1} \circ S^{\prime} \circ D^{-1} \circ B \circ C^{-1}$. Denote by $A^{\prime}=D^{-1} \circ A \circ C^{-1}$ and by $B^{\prime}=D^{-1} \circ B \circ C^{-1}$ then we get $S^{\prime-1}=A^{\prime} \circ S^{\prime} \circ B^{\prime}$.

Lemma 1 and Lemma 2 show that $\left|S E_{S}\right|$ and $\left|S E I_{S}\right|$ are constants in a given affine equivalent class, therefore we can omit the subscript $S$ when considering the class i.e. refer to $|S E|$ and $|S E I|$.

We should further check whether every self-equivalent inverse S-box gives an involution, i.e. $S^{-1}=S$ or equivalently $S \circ S=I d$.

Lemma 3. If a self-equivalent inverse $S$-box $S$ has a pair $(A, B)$, such that $A=B$ then its affine equivalent class includes an involution.

Proof. We have shown in the previous lemma that by setting $A^{\prime}=D^{-1} \circ A \circ C^{-1}$ and $B^{\prime}=D^{-1} \circ B \circ C^{-1}$ one transforms an S-box $S$ to another $S^{\prime}$. Now when $S^{\prime}$ is an involution then $A^{\prime}=B^{\prime}=I d$ and thus $A=B=D \circ C$.

Lemma 4. If an affine equivalent class, except for the class of the affine permutations, includes an involution then it includes many involutions.

Proof. If $S^{-1}=S$ then every affine permutation $C$ will give a rise to another involution $S^{\prime}=C \circ S \circ C^{-1}$.

We leave as open questions to verify that whenever we have a self-equivalent inverse in a given affine equivalent class then we have also an involution in that class; and to find a relation between the sets SE and SEI.

One approach to find an involution in a class (if it exists) is to use the brute-force method to find the inverse self-equivalence $S^{-1}=A \circ S \circ B$ through all possible pairs $(A, B)$. Instead, we can do better using Lemma 3 to look for a single affine permutation $A$, such that $S^{-1}=A \circ S \circ A$ holds. Then both $S^{\prime}=A \circ S$ or $S^{\prime \prime}=S \circ A$ are involutions. The complexity of this search is the square root of the brute-force complexity. 


\section{Conclusions}

We have shown that Shannon's expansion can be used to construct uniform sharing for certain affine equivalent classes of bijective S-boxes. We have also shown the limitations of this expansion, namely that not all 4-bit bijective S-box classes can be obtained from the 3-bit bijective S-box classes in this way.

\section{References}

1. D. Bozilov, B. Bilgin, H-A. Sahin, A Note on 5-bit Quadratic Permutations Classification, IACR ToSC 2017 (1), pp. 398-404.

2. E. Boss, V. Grosso, T. Gneysu, G. Leander, A. Moradi, T. Schneider, Strong 8-bit Sboxes with Efficient Masking in Hardware, CHES 2016, LNCS 9813, pp. 171-193.

3. B. Bilgin, S. Nikova, V. Rijmen, V. Nikov, G. Stutz, Threshold Implementations of all $3 \times 3$ and $4 \times 4$ S-boxes, CHES 2012, LNCS 7428, pp. 76-91.

4. C. De Canniere, Analysis and Design of Symmetric Encryption Algorithms, PhD thesis, KU Leuven, 2007.

5. C. Carlet, Vectorial Boolean Functions for Cryptography, chapter of the volume "Boolean Methods and Models", Cambridge University Press, Eds. Yves Crama and Peter Hammer.

6. J. Daemen, V. Rijmen, The Design of Rijndael: AES The Advanced Encryption Standard, Springer-Verlag, 2002.

7. G. Leander, A. Poschmann, On the classification of 4 bit S-boxes, WAIFI 2007, pp. $159-176$

8. R. Lidl, H. Niederreiter, Finite Fields, Encyclopedia of Mathematicsand its Applications, vol. 20, Addison-Wesley, 1983.

9. L. De Meyer, K. Varici, More Constructions for strong 8-bit S-boxes with efficient masking in hardware, 38th Symposium on Information Theory in the Benelux, 2017.

10. S. Nikova, C. Rechberger, V. Rijmen, Threshold Implementations Against SideChannel Attacks and Glitches, ICICS 2006, LNCS 4307, Springer-Verlag pp. 529-545.

11. S. Nikova, V. Rijmen, M. Schlaffer, Secure Hardware Implementation of Nonlinear Functions in the Presence of Glitches, Journal of Cryptology (2011), Volume 24, Issue 2, pp. 292-321. 